\title{
Critical Analysis of Corneal Cross-linking (Part-II): Resolving the Controversial Issues (theory versus measurements)
}

\author{
Jui-Teng Lin* \\ New Vision Inc, New Taipei City, Taiwan, ROC
}

*corresponding author: jtlin55@gmail.com

\begin{abstract}
Aims: To resolve the controversial issues of UV-light-initiated corneal collagen cross-linking $(\mathrm{CXL})$ by theoretical formulas and measured clinical outcomes.

Study design: Analysis and measured data of CXL.

Place and Duration of Study: New Vision Inc, Taipei, between June, 2021 and August 2021.

Methodology: The controversial issues are addressed and resolved by analytical formulas including: the validation of Bunsen Roscoe law (BRL), the cutoff light intensity, the minimum corneal thickness, the demarcation line depth, the role of oxygen riboflavin (RF) concentration. The overall CXL efficacy is governed by UV-A light intensity, dose, exposure time, mode of exposure (pulsed or $\mathrm{CW}$ ), riboflavin concentration, diffusion and drops pre-operation and interoperation administration, concentration of oxygen in the stromal tissue (pre-op and inter-op), and environmental conditions. The length of the riboflavin presoaking time and viscosity of the riboflavin film also affect the crosslink depth. Analytic formulas are derived for the scaling laws for type-I and type-II efficacy, given by the square-root of light intensity, and light dose, respectively. Conclusion: The controversial issues of CXL may be partially resolved via analytic formulas, and compared with measurements. The scaling laws of type-I and type-II efficacy are different and given by analytic formulas. Our formulas also predict the maximum light intensity and the minimum corneal thickness, which are consistent with measurements.
\end{abstract}

Keywords: corneal collagen crosslinking, CXL, efficacy, riboflavin, modeling, safety dose, ultraviolet light.

\section{Introduction}

UVA light (at $365 \mathrm{~nm}$ ) and riboflavin photosensitized corneal collagen cross-linking $(\mathrm{CXL})$ was first proposed by Spoerl et al [1,2] in1998 to increase the corneal biomechanical strength and stabilize the ectatic cornea. The standard Dresden (SD) protocol was proposed (in 2003) by Wollensak et al [3], in which a UVA light intensity of $3.0 \mathrm{~mW} / \mathrm{cm}^{2}$ was applied to the cornea for an irradiation time of 30 minutes, such that a light fluence (dose) of $5.4 \mathrm{~J} / \mathrm{cm}^{2}$ was delivered to the cornea. To shorten the irradiation time of the SD protocol, accelerated CXL (AC) was also developed [4,5], based on Bunsen and Roscoe law [6], leading to the AC protocol given by light intensity of $I=(3,9,18,30,45) \mathrm{mW} / \mathrm{cm}^{2}$, with the associated irradiation time is inverse proportional to the light intensity given by $\mathrm{t}=(30,10,5,3,2)$ minutes, such that the total dose applied to the cornea is fixed at $5.4 \mathrm{~J} / \mathrm{cm}^{2}$ [1]. In the past 18 years (2003-2020], over 1,000 articles were published on the clinical aspects of CXL. In comparison, only about 20 articles were published regarding to the basic kinetics and/or the theory of CXL [7-27], in which over $75 \%$ was published by Lin et al [7-23]. The lack of in-depth fundamental studies and lack of conclusive theoretical predictions, the following controversial (clinical/theoretical) issues remained to be resolved [15,22]:

(a) Validation of standard Dresden (SD) protocol for the minimum (safety) corneal thickness of $400 \mathrm{um}$, and a required light dose of $5.4 \mathrm{~J} / \mathrm{cm}^{2}$ proposed by Spoerl et al [1-3].

(b) The threshold damage dose of endothelium $\left(0.63 \mathrm{~J} / \mathrm{cm}^{2}\right)$ proposed by Wollensak et al [28] was largely underestimated, comparing to the more recent data of Mooren et al [29]; and 
leading to an overestimated minimum thickness of 400 um;

(c) Validation of the Bunsen and Roscoe law (BRL) of reciprocity [27] for accelerated CXL (AC) protocol.

(d) For a fixed light dose, AC was clinically reported to be less efficient than SD [4,5], and having a maximum light intensity limitation [30];

(e) Improved CXL efficacy and the role of oxygen [31-34];

(f) Improved efficacy by pulsed light [35-37];

(g) Improved efficacy by higher riboflavin concentration [17,18,38-41];

(h) Validation of SD based on a dose of $5.4 \mathrm{~J} / \mathrm{cm}^{2}$, comparing to the recent clinical data of King et al [42] using a lower dose $\left(2.7 \mathrm{~J} / \mathrm{cm}^{2}\right)$, which is half of SD.

(i) New criteria for minimum corneal thickness $\left(Z^{*}\right)$, in which sub400um thin corneas (214 398 um) was reported by Hafez et al [43];

(j) Factors influencing the demarcation line depth (DLD)

(k) Comparing the Theory/formulas of $Z^{*}$ and DLD, developed by Lin [12,23], Kling et al [27] and Mazzotta et al [44].

(I) The new efficacy scaling law developed by Lin, a nonlinear law replacing the linear law based on BRL;

(m) Strategies for improved CXL efficacy and optimal protocols.

The present article intends to resolved the above listed issues totally (if theoretically), and partially (if by experimental evidences). Comprehensive and updated formulas will be used to analyze, support, or predict the recently reported clinical outcomes including that of Hafez et al [43] regarding minimum cornea thickness, demarcation line depth; the role of riboflavin concentration reported by O'Brart et al [41]. Formula for cut-off light intensity is derived and compared with the reported data by Wernli et al [30]. New scaling laws for CXL efficacy for both type-I and type-II will be developed. Analytic formulas for CXL efficacy is up-dated for the influencing factors of oxygen, riboflavin concentration, light intensity, light dose, corneal thickness, and kinetic rate constants.

\section{Methods and Theory}

\subsection{Crosslink Rate Equation}

Using the short hand notations: $\mathrm{C}$ and $\mathrm{T}$ for the RF ground and excited triplet state; $\mathrm{R}$ for the active radical, $\mathrm{S}$ for the singlet oxygen $\left[{ }^{1} \mathrm{O}_{2}\right] ; \mathrm{X}$ for the ground state oxygen $\left[{ }^{3} \mathrm{O}_{2}\right]$; and $[\mathrm{A}]$ for the available extracellular matrix substrate, the crosslink rate equation is given by $[22,23]$

$$
\frac{\mathrm{d}[\mathrm{A}]}{\mathrm{dt}}=-\left(\mathrm{K}_{3} \mathrm{~T}+\mathrm{K}_{1} \mathrm{R}+\mathrm{K}_{2} \mathrm{~S}\right)[\mathrm{A}]
$$

Eq. (1) includes three crosslink pathways: (i) the type-I direct coupling of $T$ and the substrate $[A]$; (ii) and the coupling of the radical $(R)$ and $[A]$; and (iii) the oxygen-mediated, type-II term due to the singlet oxygen coupling with [A]. Both type-I and type-II pathway can occur simultaneously, and the ratio between these processes depends on the type of photosensitizers (PS) used, the concentrations of PS, substrate and oxygen, the kinetic rates involved in the process, and the light intensity, dose, PS depletion rate etc.

We note that Eq. (1) is much more complete than previous models of Kamave et al [24], which is the special case when $\mathrm{C}(\mathrm{t}$ ) is a constant (using a continuing resupply of $\mathrm{RF}$, or for the case of a perfect regeneration of RF). They also ignored the type-I major term of $K_{1} R$. Schumacher et al 25] and Semchishen et al [26] are limited to type-I conversion, $K_{1} R$, and ignored $k_{3} T$ term and the oxygen-mediated term, $\mathrm{K}_{2} \mathrm{~S}$ in our Eq. (1). They also ignored the RGE cycle effects. The modeling of Kling et al [27] is based on Kamave [24], but only showed the algorithm for numerical calculations without analytic formulas. Comparing to the above described previous modeling [24- 
27], our modeling, shown by Eq. (1), is the most complete and accurate one.

The quasi-steady-state solutions are given by [21,22]: $T=b \operatorname{lgC}, S=g^{\prime} k_{4} T X$; with $g=1 /\left(k^{\prime \prime}+\right.$ $\left.K_{3}[A]+k_{4} X\right) ; g^{\prime}=1 /\left(k_{6}+k_{1} C+K_{2}[A]\right)$. But radical $(R)$ is more complex given by the solution of $[22,23]$

$$
k^{\prime} R^{2}+G R-H=0
$$

where where $\mathrm{G}=\mathrm{k} " \mathrm{X}+\mathrm{K}_{1}[\mathrm{~A}]$ and $\mathrm{H}=\mathrm{K}_{3}[\mathrm{~A}] \mathrm{T}$; with $\mathrm{T}=\mathrm{blgC}$.

Eq. (2) has two special cases: Case (i) for unimolecular termination dominant, or $G>>k^{\prime} H, R=$ $\mathrm{K}_{3}(\mathrm{blgC}[\mathrm{A}] / \mathrm{G})(1-0.5 \mathrm{H} / \mathrm{G})$, which is a linear increasing function of $\mathrm{H} / \mathrm{G}$, and there is an oxygen inhibition $(\mathrm{OIH})$ effect which reduces the radical $(R)$ and the efficacy, because $G$ is an increasing function of oxygen (or X).

Case (ii) for bimolecular termination dominant, with $H \gg>G R$, we obtain, $R=\left[H / k^{\prime}\right]^{0.5}$, which is a nonlinear function of $\left[\mathrm{K}_{3}(\mathrm{blgC})[\mathrm{A}]\right]^{0.5}$, a square root function. In contrast to case (i), the OlH plays minor role in case (ii) via the oxygen-dependence of $\mathrm{g}$.

\subsection{The Efficacy formulas}

For type-I dominant case, from Eq. (1), with $\mathrm{K}_{2} \mathrm{~S}=0$, and for bimolecular termination dominant, we obtain, $R=\left[K_{3}[A] T / k^{\prime}\right]^{0.5}$. with $T=\operatorname{blgC}(t)$. For $g$ is approximated as $g=1 /\left(K_{3}[A]\right), R$ becomes $R=\left[T^{\prime} / k^{\prime}\right]^{0.5}$, with $T^{\prime}=b I C(t)$, we obtain

$$
\frac{\mathrm{d}[\mathrm{A}]}{\mathrm{dt}}=-\mathrm{T}^{\prime}+\mathrm{K}_{1} \sqrt{\mathrm{T} / k^{\prime}}[\mathrm{A}]
$$

Considering a non-perfect regeneration case with $C(t)=C_{0} \exp \left(-d^{\prime} t\right)$, with $d^{\prime}=b l g "$, and g" defining the degree of regeneration (with $g^{\prime \prime}=0$, for the perfect case). the approximated analytic solution of Eq. (3) leads to

$$
\left.\mathrm{CE}=1-\left[1+\mathrm{k} \mathrm{H}^{\prime}\right)\right] \exp (-\mathrm{H})
$$

where $k=b I C_{0} / A_{0}$ is the contribution from the $K_{3} T$ term; $H(t)=2 d\left[1-\exp \left(-0.5 d^{\prime} t\right)\right] / d^{\prime}, H^{\prime}(t)=[1-\exp (-$ $\left.\left.0.5 \mathrm{~d}^{\prime \prime} \mathrm{t}\right)\right] / \mathrm{d}^{\prime}$, with $\mathrm{d}=\mathrm{K}_{1}\left(\mathrm{blC}_{0} / \mathrm{k}^{\prime}\right)^{0.5}, \mathrm{~d}^{\prime}=\mathrm{blg}$ " and $\mathrm{d}^{\prime \prime}=\mathrm{d}^{\prime}-0.5 \mathrm{~d}$. Eq. (4) has transient state, $\mathrm{H}(\mathrm{t})=2 \mathrm{dt}$, $\mathrm{H}^{\prime}=0.5 \mathrm{~d}$ "t. The steady state with $\mathrm{H}=2 \mathrm{~d} / \mathrm{d}^{\prime}=2 \mathrm{~K}_{1}\left[\mathrm{C}_{0} /\left(\mathrm{bgk}^{\prime} \mathrm{I}\right)\right]^{0.5}$, and $\mathrm{H}^{\prime}(\mathrm{t})=1 / \mathrm{d}^{\prime \prime}$, which is proportional to $\left[\mathrm{C}_{0} /(\mathrm{bl})\right]^{0.5}$, an increasing function of $\mathrm{C}_{0}$, but decreasing function of the light intensity, $\mathrm{I}(\mathrm{z})=\mathrm{I}_{0}$ $\exp \left(-\mathrm{A}^{\prime} z\right)$. Therefore, higher light intensity leads to lower steady state conversion than that of lower light intensity. This feature will be shown later in Fig.3, in comparing to type-II. We note that the OlH effect plays no role in this case (ii) of type-I process. This steady state feature may be also used to analyze the maximum light intensity feature clinically measured by of Wernli et al [14], where a sudden efficacy decrease at high light intensity (about $65 \mathrm{~mW} / \mathrm{cm}^{2}$ ).

For type-Il dominant case, we need to solve for oxygen concentration, $\mathrm{X}(\mathrm{t})$, given by the kinetic equation [22]

$$
\frac{\mathrm{dX}}{\mathrm{dt}}=-\left(\mathrm{k}^{\prime \prime} \mathrm{R}+\mathrm{k}_{4} \mathrm{~T}\right) \mathrm{X}+\mathrm{k}_{6} \mathrm{~S}+\mathrm{P}
$$

For the case that there is no external resupplied oxygen (or $\mathrm{P}=0$ ), and using the first-order solution with $[A]=A_{0}$ in the function of $g$ and $S=k_{4} g^{\prime} T X$, with $T=T^{\prime} /\left(K_{3} A_{0}\right)$, and a time-independent $g^{\prime}=1 /\left(k_{6}+k_{1} C_{0}+K_{2} A_{0}\right)$, we obtain $[22,23]$

$$
\begin{aligned}
& \mathrm{X}(\mathrm{t})=\mathrm{X}_{0} \exp [-\mathrm{Dt}] \\
& \mathrm{D}=\mathrm{k}_{4}\left(1-\mathrm{g}^{\prime}\right) \mathrm{T}^{\prime}+\mathrm{k}^{\prime \prime} \sqrt{\mathrm{T}^{\prime} / k^{\prime}}
\end{aligned}
$$

Time integral of Eq. (1) (for $K_{1}=K_{3}=0$ ), only the $K_{2} S$ term, with $S=\left(k_{4} / K_{6}\right) g^{\prime} T X(t)$ and $X(t)$ given by Eq. (6), we obtain

$$
\mathrm{CE}=\mathrm{p}^{\prime}[1-\exp (-\mathrm{Dt})] / \mathrm{D}
$$


where $p^{\prime}=\left(k_{4} K_{2} /\left(k_{6} K_{3}\right)\left(g^{\prime} T^{\prime} X_{0}\right)\right.$, and $T^{\prime}=b I C_{0}$. Above CE has a transient state $C E=p^{\prime} t$, which is proportional to the light dose $\left(\mathrm{I}_{0} \mathrm{t}\right)$, but a steady state $C E=p^{\prime} / \mathrm{D}$, which is almost independent to light intensity. Our Eq. (6) and (7) may be compared with the Eq. (5) and (11) of Kling et al [5], however their formulas are not expressive forms, including unknown parameter [EM] in the equations and can be solved only numerically. They also ignored the type-l process, and assumed the oxygen-mediated type-II is dominant.

\subsection{The maximum light intensity}

The maximal (or cutoff) light intensity reported by Wernli et al [30] may be analyzed by the following. We will use type-I efficacy, Eq, (4), let the steady-state $C E$ equals a minimum $C E=E_{T H}$, a steady-state threshold CE for sufficient CXL. Solving for $2 d / d^{\prime}=\ln \left[(1+k / d ") /\left(1-E_{T H}\right)\right]$, we obtain the cutoff maximum intensity $I_{0}^{*}$ (on surface, $z=0$ ) given by (for $k / d "<<1$ ),

$$
I_{0}{ }^{*}=\left[\mathrm{G}^{\prime} / \ln \mathrm{E}^{\prime \prime}\right]^{2}
$$

where $G^{\prime}=2 K_{1}\left(b C_{0} / k^{\prime}\right)^{0.5} /(b g ")$, and $E^{\prime \prime}=1 /\left(1-E_{T H}\right)$. Above formula defines the cutoff light intensity proportional to the ratio of $\mathrm{C}_{0}$ and $\mathrm{InE}^{\prime}$. For a given $\mathrm{C}_{0}=0.1$ to $0.4 \%$, the predicted range of $\mathrm{I}_{0}{ }^{*}$ is about 45 to $65 \mathrm{~mW} / \mathrm{cm}_{2}$, to be shown later.

\subsection{Minimum thickness}

To estimate the safety dose and minimum thickness, we need to find the time $(t)$ and depth (z) dependence RF concentration, C(t,z), and light intensity, I $(\mathrm{z}, \mathrm{t})$. Lin [11] has developed a timedependent Beer Lambert law [ ] for the light intensity given by $I(z, t)=I_{0} \exp \left[-\left(q^{\prime}-B t\right) z\right]$, with $q^{\prime}=2.3\left(a^{\prime} C_{0}+Q\right)$ is an effective absorption constant and $B=2.3 C_{0}\left(a^{\prime}-b^{\prime}\right) d^{\prime}$, and an approximated $C(z, t)=C_{0} \exp \left(-d^{\prime} t\right)$, with $d^{\prime}=b l g ' t$. Where $a^{\prime}=204(1 / \% / c m)$ and $b^{\prime}$ (unknown value) are the extinction coefficients of RF and the photolysis product, respectively; $Q=13.9(1 / \mathrm{cm})$ is the absorption coefficient of the stroma.

The light dose $(E)$ at a given stroma depth $(z)$ can be easily found by the time integral of $I(z, t)$ leading to $E(z, t)=I_{0} G(t) \exp \left(-q^{\prime} z\right)$, with $G(z, t)=[1-\exp (-B z t)] /(B z)$. The corneal minimum thickness $\left(Z^{*}\right)$, defined by a damage dose threshold value of $E^{*}$ (or the safety dose) may be obtained by $E(z)=E^{*}$, and solve for $z=Z^{*}$. For small Bzt, $E^{*}=E_{0}(1-0.5 B z t) \exp \left(-q^{\prime} z\right)$, which leads to the minimum corneal thickness given by

$$
Z^{*}=\left(1 / \mathrm{q}^{\prime}\right) \ln \left[\left(\mathrm{E}_{0} / E^{*}\right)\left(1-\mathrm{B} Z^{*} \mathrm{t}\right)\right]
$$

which has an analytic solution, when $B z t=0$. For small Bzt, $\ln (1-B z t)=-B z t$, Eq. (9) leads to $\left.Z^{*}=\left(1 / q^{\prime}\right) \ln (R) /[1+B t)\right] R=E_{0} / E^{*}$, which is time dependent due to the depletion of $C(t)$, and needs numerical calculation for $Z^{*}$ vs. light dose. We note that $Z^{*}=\left(1 / q^{\prime}\right) \ln (R)$, for the constant concentration case (with $B=0$ ). We also note that $q^{\prime}=2.3\left(a^{\prime} C_{0}+Q\right.$ ) is a decreasing function of $R F$ concentration, $\mathrm{C}_{0}$.

\subsection{Demarcation line depth}

The demarcation line depth (DLD) may be defined by when the conversion efficacy (CE) is larger than an efficacy threshold value (E') for collagen tissue to be effectively affected to form the DLD. For type-I of Eq. (4), the transient state, $\mathrm{H}(\mathrm{t})=2 \mathrm{dt}$, with $\mathrm{d}=\mathrm{K}^{\prime} \mathrm{I}_{0}{ }^{0.5} \exp \left(-0.5 \mathrm{q}^{\prime} \mathrm{z}\right), \mathrm{K}^{\prime}=\mathrm{K}_{1}\left[\mathrm{bC} \mathrm{C}_{0} / \mathrm{k}^{\prime}\right]^{0.5}$, and ignored $\mathrm{kH}^{\prime}<<1$, and let $\mathrm{CE}=\mathrm{E}^{\prime}$, or $\mathrm{H}=\ln \left[1 /\left(1-\mathrm{E}^{\prime}\right)\right]$, we obtain (for type-I)

$$
\mathrm{Z}^{\prime}=\left(2 / \mathrm{q}^{\prime}\right) \ln \left(K E_{0} / \ln E^{\prime \prime}\right)
$$

with $q^{\prime}=2.3\left(a^{\prime} C_{0}+Q\right), K=2 K^{\prime} I_{0}^{-0.5}$, and $E^{\prime \prime}=1 /\left(1-E^{\prime}\right)$, which has a similar tend as that of $Z^{*}$, both are increasing function of $\ln \left(E_{0}\right)$, but decreasing function of the damage threshold dose $\left(E^{\prime}\right)$. However, $Z^{\prime}$ is also inverse proportional to the square root of light intensity (for a given light dose). That is higher intensity has a smaller DLD, in consistent with the measured data [ ]. We may rewrite Eq. (10) as two parts: $Z^{\prime}=Z 1-Z 2$, with $Z 1$ (for $E_{0}$ dependent only) $=\left(2 / q^{\prime}\right) \ln \left[2 K^{\prime} E_{0} / \operatorname{lnE} E^{\prime \prime}\right.$, and $Z 2$ (for only intensity function $)=\left(1 / q^{\prime}\right) \ln \left(I_{0}\right)$, which is proportional to light intensity. Given a value of $q^{\prime}=0.01$ (1/um), and let $G=2 K_{1}\left[b_{0} / k^{\prime}\right]^{0.5}\left(E_{0} / I n E "\right)$, Eq. (10) becomes 


$$
\mathrm{Z}^{\prime}=200 \ln \left(\mathrm{G} / \sqrt{I_{0}}\right)
$$

for $I_{0}$ in $\mathrm{mW} / \mathrm{cm}_{2}$. and $Z^{\prime}$ in um.

Similarly, for type-II, using the transient state, $C E=p^{\prime} t=K^{\prime \prime \prime} I_{0} \quad \exp \left(-q^{\prime} z\right)$, with $\mathrm{K}^{\prime \prime}=\mathrm{k}_{4} \mathrm{~K}_{2} /\left(\mathrm{k}_{6} \mathrm{~K}_{3}\right)\left(\mathrm{g}^{\prime} \mathrm{bC} \mathrm{C}_{0} \mathrm{X}_{0}\right)$, we obtain (for type-II)

$$
\mathrm{Z}^{\prime}=\left(1 / \mathrm{q}^{\prime}\right) \ln \left(K E_{0} / \ln \mathrm{E}^{\prime \prime}\right)
$$

with $\mathrm{K}=\mathrm{k}_{4} \mathrm{~K}_{2} /\left(\mathrm{k}_{6} \mathrm{~K}_{3}\right)\left(\mathrm{g}^{\prime} \mathrm{bC} \mathrm{C}_{0}\right)$, and it is proportional to the light dose $\left(\mathrm{E}_{0}\right)$, unlike that of type-l.

As expected, Eq. (10) and (11) for the $Z^{\prime}$ (or DLD) have the similar trend as that of $Z^{*}$, Eq. (10), both are increasing function of $\ln \left(E_{0}\right)$. We may rewrite $Z^{\prime}=\left(1 / q^{\prime}\right) \ln R^{\prime}$, with $R^{\prime}=K E_{0} / \ln E^{\prime \prime}$, with $\mathrm{K}=\mathrm{K}^{\prime}$ for type-I, and $\mathrm{K}=\mathrm{K}^{\prime \prime}$ for type-II. The actual value of $\mathrm{Z}^{\prime}$ may be calculated if the $\mathrm{E}^{\prime}$ value can be measured accurately at a reference point.

\subsection{Scaling laws}

From our theoretical formulas, we summarize the following important scaling laws, which give us the role of each of the influencing factors and their nonlinear relationships.

(i) Efficacy of type-I CXL given by Eq. (4), has a transient state of $C E=1-\exp (-X)=X t(1-0.5 X t$ $+.$.$) , with X=m\left[C_{0} l_{0}\right]^{0.5}$, and a fit constant $m=8$ to 12 ; Therefore, $C E$ is a nonlinear function of $X$ having a scaling law of

$$
\mathrm{Xt}=\mathrm{m}\left[\mathrm{C}_{0} \mathrm{I}_{0}\right]^{0.5 \mathrm{t}}=\mathrm{E}_{0}\left[\mathrm{C}_{0} / \mathrm{I}_{0}\right]^{0.5}
$$

with a scaling power of -0.5 for light intensity and a linear power of dose $\left(E_{0}\right)$.

In comparison, steady state $C E=1-\exp \left(-Y^{0.5}\right)=Y\left(1-Y / 2+Y^{2} / 6+..\right)$, with

$$
\mathrm{Y}=\mathrm{m}^{\prime}\left[\mathrm{C}_{0} / \mathrm{I}_{0}\right]^{0.5}
$$

which has a scaling power of -0.5 for light intensity, same as Eq. (12.a), but independent to $\mathrm{E}_{0}$ (or dose).

(ii) For type-II $C X L$, given by Eq. (10), has a transient state of $C E=1-\exp \left(-X^{\prime} t\right)$, with $X^{\prime}=$ $\mathrm{m}^{\prime}\left[\mathrm{C}_{0} \mathrm{l}_{0} \mathrm{X}_{0}\right]$, having a linear scaling law of

$$
X^{\prime} t=C_{0} E_{0} X_{0}
$$

which is proportional to the light dose and oxygen initial concentration $\left(\mathrm{X}_{0}\right)$. In comparison, steady state $\mathrm{CE}=1-\exp \left(-\mathrm{KX}_{0}\right)$, which is almost independent to the light dose, or RF concentration and it is solely depending on oxygen initial concentration $\left(\mathrm{X}_{0}\right)$, given by a scaling law of

$$
\mathrm{CE}=\mathrm{KX}_{0}\left(1-0.5 \mathrm{KX}_{0}+\mathrm{KX} \mathrm{X}_{0} / 6+\ldots\right)
$$

(iii) Scaling law for minimum corneal thickness, from Eq. (9),

$$
Z^{*}=\left(1 / q^{\prime}\right) \ln (R) \text {, with } R=E_{0} / E^{*}
$$

(iv) Scaling law for demarcation line depth (DLD) is given by, from Eq. (10.a) for type-I

$$
Z^{\prime}=\left(2 / q^{\prime}\right) \ln \left(K E_{0} l_{0}^{-0.5}\right)
$$

and from Eq. (11) for type-II,

$$
Z^{\prime}=\left(1 / q^{\prime}\right) \ln \left(K^{\prime} E_{0} X_{0}\right)
$$


We note that both $Z^{*}$ and $Z^{\prime}$ have similar functional form proportional to In $(R)$, with $R$ proportional to the light dose $\left(E_{0}\right)$.

\section{Results and Discussions}

\subsection{Analysis of maximum (cutoff) light intensity (MLI)}

A maximum (cutoff) light intensity (MLI) was reported by Wernli et al [30] that he efficacy of CXL shows a sudden decrease with very high intensity UV light. This feature may be analyzed by our theoretical curve in Fig. 1 for efficacy versus light intensity showing the the maximum (cutoff) light intensity (MLI) defined by the threshold efficacy $\left(E_{T H}\right)$ based on formula for the steady state $\mathrm{CE}$ of Eq. (4), given by $C E=1-\exp \left(-\mathrm{G}^{\prime} \mathrm{I}_{0}^{-0.5}\right)$. MLI is calculated as 50 to $70 \mathrm{~mW} / \mathrm{cm}^{2}$, for $\mathrm{G}^{\prime}=5$ to 6 ; in consistent with the data of Wernli et al [30], about 45 to $55 \mathrm{~mW} / \mathrm{cm}^{2}$.

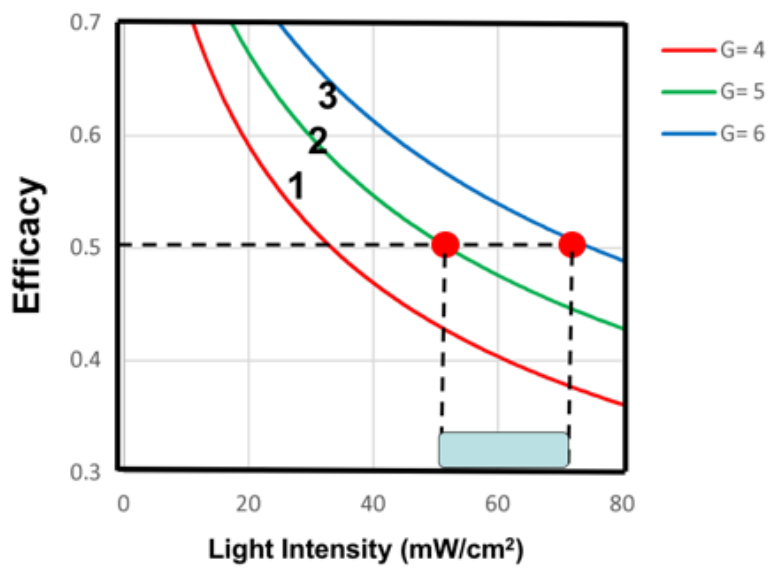

Fig. 1 Calculated efficacy versus light intensity showing the the maximum (cutoff) light intensity (MLI), for threshold efficacy $\left(E_{T H}\right)$ of $50 \%$, and $G^{\prime}=(4,5,6)$ for curves $(1,2,3)$ based on $C E=1-\exp (-$ $\mathrm{G}^{\prime} \mathrm{I}^{-0.5}$ ).

\subsection{Analysis of thin corneas}

The recent clinical works of Hafez et al [43] showed safety cases for very thin corneas of (214 to $390 \mathrm{um}$ ), much less than the conventional minimum criterion of 400 um (based on the Dresden protocol). Our formula demonstrates the theoretical minimum thickness $\left(Z^{*}\right)$ could be as thin as $100 \mathrm{um}$ (after epithelium removed), as far as the applied dose $\left(E_{0}\right)$ is less than the threshold value $\left(E^{*}\right)$. For example, for the case of $B z t=0$, Eq. (9) becomes $Z^{*}=\left(1 / q^{\prime}\right) \ln (R)$, with ratio $\mathrm{R}=\mathrm{E}_{0} / \mathrm{E}^{*}$. For example, for $\mathrm{C}_{0}=0.2 \%$, $\mathrm{a}^{\prime}=204(1 / \% / \mathrm{cm}), \mathrm{Q}=13.9(1 / \mathrm{cm})$, we obtain $q^{\prime}=469 C_{0}+32=79(1 / \mathrm{cm})=0.0079(1 / \mathrm{um}), 1 / q^{\prime}=127$ (um), for $C_{0}=0.1 \%$, and $Z^{*}=127 \ln (R)$. Using $Z^{*}=127$ um for $R=2.72$, as the reference, then the safety thickness is given by $Z^{*}=127 \ln \left(R^{\prime}\right)=(127,203,229,230,292,380,432,556) \quad u m, \quad$ for $R=(2.72,5,10,20,30,55,80)$ and $\ln R=(1 \cdot 0,1 \cdot 6,1 \cdot 8,2 \cdot 3,3 \cdot 0,3 \cdot 4,4 \cdot 0,4 \cdot 38)$, In The thin corneal thickness of Hafez et al [43] at $z=214$ um, corresponding to our $R=8$, or a dose of $E_{0}=8 E^{*}$; and $z=400 u m$, for $R=45$. However, the actual value of $E^{*}$ and the referenced ratio $\left(R^{\prime}\right)$ require further clinical measurements. Hafez et al [43] reported the non-linear relation between the UV irradiation time and predicted demarcation line (referred to their Figure 3 and Table 2). Fig. 2 shows calculated curves based on $Z^{*}=$ $\left(1 / q^{\prime}\right) \ln (R)$, with $q^{\prime}=2.3\left(a^{\prime} C_{0}+Q\right)$ is a decreasing function of RF concentration, $C_{0}$. That is, higher concentration has a better safety protection such that thinner cornea is allowed. In contrast, higher dose (or larger $R=E_{0} / E^{*}$ ) requires a thicker cornea. For example, for $Z^{*}=\left(16.5 / C_{0}\right) \ln (R)$, for $R=12, Z^{*}=(100,200,400)$ um, for $C_{0}=(0.1,0.2,0.4) \%$. Our $Z^{*}$ formula is more accurate and expressive (in analytic form) than that of Kling et al [27] which showed complex algorithm requiring numerical solution, and was limited to type-II process. 


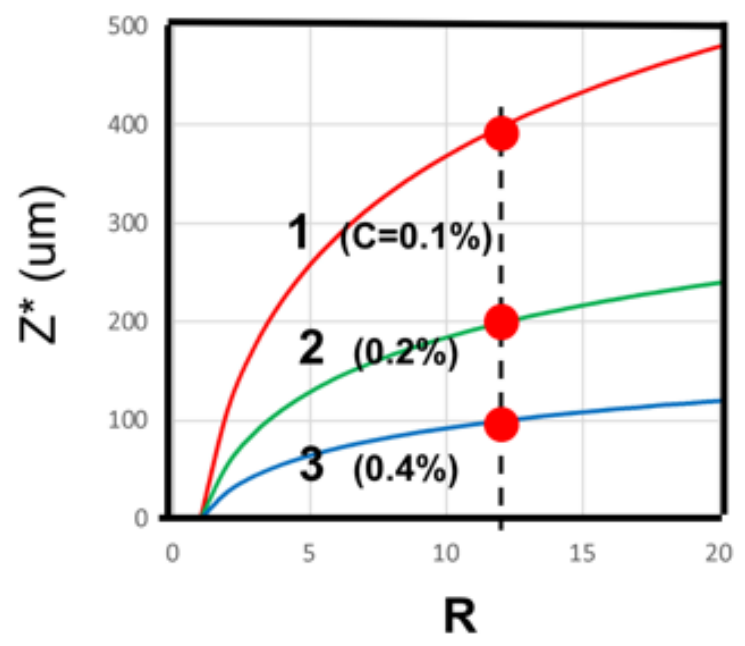

Fig. 2 Minimum corneal thickness $\left(Z^{*}\right)$ versus threshold ratio $R=E_{0} / E^{*}$, based on $Z^{*}=\left(1 / q^{\prime}\right) \ln (R)$, for RF concentration, $\mathrm{C}_{0}=(0.1,0.2,0.4) \%$, for curves $(1,2,3)$.

\subsection{Analysis of demarcation line depth (DLD)}

Fig. 3 shows the DLD ( $\left.Z^{\prime}\right)$ versus light intensity based on Eq. (10.b), with threshold ratio factor $G=K=2 K^{\prime} E_{0} / l n E "$, which is proportional to the ratio of light dose and efficacy threshold $(E ")$, and $K^{\prime}=K_{1}\left[b_{0} / k^{\prime}\right]^{0.5}$, or RF concentration. Fig. 3 shows that $Z^{\prime}=(100,180,230)$, for $G=(10,15,20)$ at a fixed light intensity of $40 \mathrm{~mW} / \mathrm{cm}^{2}$. Fig. 4 shows $D L D$ versus $G, Z^{\prime}=(130,200,300)$ um for a given $\mathrm{G}=15$ and various intensity $\mathrm{I}=(10,30,60) \mathrm{mW} / \mathrm{cm}^{2}$. Both Figures show that $D L D$ is decreasing function of light intensity, but increasing function of $G$ factor, noting hat $G$ is proportional to the light dose $E_{0}$. Fig. 5 compares the DLD from measured data of Hafez et al [43], and calculated values based Eq. (10.b). They also showed the $Z^{*}$ vs. UV irradiation time (t), or dose for a fixed light intensity. However, their data were based on the Dresden protocol of $t=30$ minutes, for 3 $\mathrm{mW} / \mathrm{cm}^{2}$ intensity, and an under-estimated damage dose threshold $\left(E^{*}\right)$. Therefore, our formula based on the relative ratio of $E_{0} / E^{*}$ is much more accurate (if $E^{*}$ could be measured accurately). Figure 3 and Table 2 of Hafez et al [43] may be compared with our formula, Eq. (10), $Z^{*}=\left(1 / q^{\prime}\right) \ln \left(K E_{0} / \operatorname{lnE}\right)$, which is proportional to $\ln$ (irradiation time), for a fixed light intensity $\left(I_{0}\right)$, and it is an increasing function of $\ln \left(E_{0}\right)$ showing a consistent trend as their Table 2 and Figure 3.

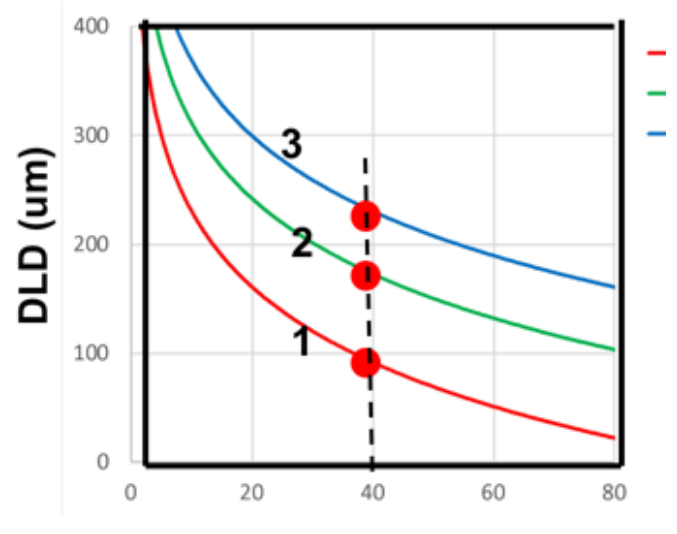

Light Intensity $\left(\mathrm{mW} / \mathrm{cm}^{2}\right)$ 
Fig. 3 Demarcation line depth (DLD) versus light intensity for various threshold ratio factor $G=(10$, $15,20)$, for curves $(1,23)$.

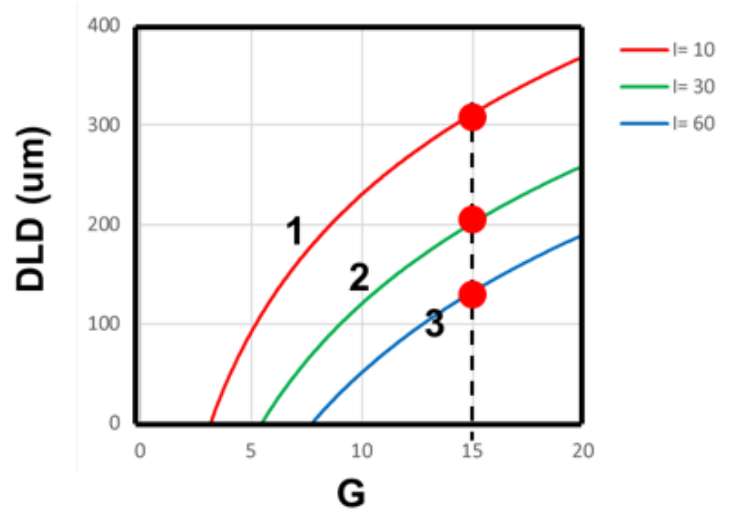

Fig. 4 Demarcation line depth (DLD) versus threshold ratio factor $G$, for intensity $I=(10,30,60)$ $\mathrm{mW} / \mathrm{cm}^{2}$, for curves $(1,23)$.

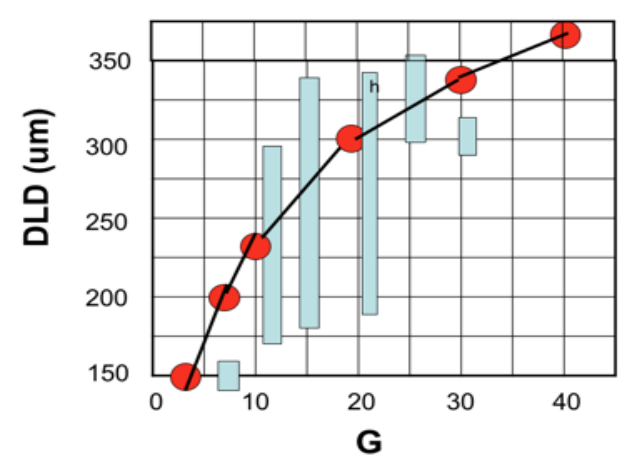

Fig. 5 Demarcation line depth (DLD) versus threshold ratio factor $G$, for intensity $\mathrm{I}=10 \mathrm{~mW} / \mathrm{cm}^{2}$, where bars are measured data of Hafez et al [43], and red dots are calculated.

\subsection{Role of RF concentration}

O'Brart et al [41] reported that efficacy is an increasing function of RF concentration (in the stroma). Clinical studies of Lang et al [5] showed that AC had less efficacy than standard low intensity $\left(3 \mathrm{~mW} / \mathrm{cm}^{2}\right) \mathrm{CXL}$ for the same fluence (dose) based on BRL. To overcome this intrinsic drawback of $A C$, Lin $[16,20]$ recently proposed a new protocol called riboflavin concentration controlled method (CCM) to improve the efficacy of AC by supplemental RF during the UV exposure to compensate the fast depletion of RF by UV light. However, RF concentration, $C(t)$, may be partially self-compensated due to the regeneration effect $(R G E)$, in which $C(t)=$ $C(z, t)=C_{0} \exp (-b l g " t)=C_{0}$, for a perfect RGE case with $g^{\prime \prime}=0$, which also depends on the type of photoinitiator. The optimal concentration was also theoretically proposed, specially for the efficacy in the stroma (with $z>0$ ) [11]. From Eq. (4), the type-I steady state efficacy given by $\mathrm{H}=$ $2 \mathrm{~K}_{1}\left[\mathrm{C}_{0} /\left(\mathrm{bgk}^{\prime} \mathrm{l}\right)\right]^{0.5}$, which is an increasing function of $\left[\mathrm{C}_{0} / \mathrm{l}_{0}\right]^{0.5}$. To show this scaling law, Fig. 6 
shows the calculated efficacy curves based on a simplified form of Eq. (4), CE=1-exp(-m[C $\left.\left.\mathrm{C}_{0} / \mathrm{l}_{0}\right]^{0.5}\right)$, with $\mathrm{m}=10$ as a fit parameter comparing to measured data. For a fixed light intensity, efficacy is an increasing function of $\mathrm{C}_{0}{ }^{0.5}$, but a decreasing function of $\mathrm{I}_{0}{ }^{0.5}$. For examples, (shown by the red lots of Fig.6), efficacy $C E=(0.8,0.7,0.6)$, for $I=(9,18,30) \mathrm{mW} / \mathrm{cm}^{2}$, at a given $\mathrm{C}_{0}=0.25 \%$. The measured data of O'Brart et al [41] are fit to our calculated curve.

As discussed in our previous paper [22], $\mathrm{C}(\mathrm{t})$ is a constant if there is a continuing resupply of $\mathrm{RF}$, or for the case of a perfect regeneration (RGE) of RF (or a catalytic cycle). To demonstrate the RF depletion, we conducted a measurement of RF (mixed with pure water) under UV irradiation at various RF concentrations, in which the increase of light intensity passing through the RF solution (path of $10 \mathrm{~mm}$ ) was recorded indicating the decrease of RF which also has color changes. This feature can be explored by $I(z, t)=I_{0} \exp \left[-q^{\prime}+B t\right] z$, a revised time-dependent Beer Lambert law $[11,17\}$, with $B=2.3\left(a^{\prime}-b^{\prime}\right) C_{0} d^{\prime}$, proportional to the depletion rate of $R F\left(d^{\prime}\right)$ and its initial concentration $C_{0}$. If $d^{\prime}=0$, then $I(t, z)$ is time independent. As shown by Fig. 7 , high $R F$ concentration has a larger light intensity increasing slope, defined by $B$.

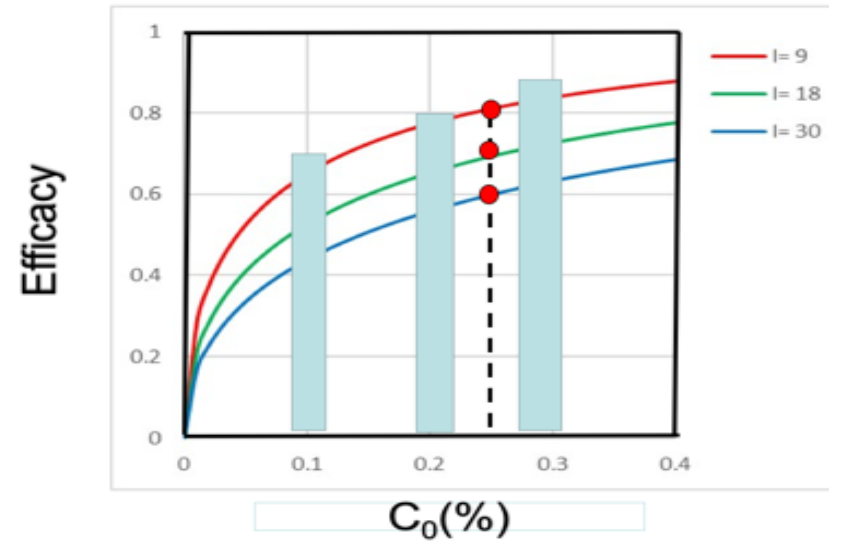

Fig. 6 Calculated efficacy versus RF concentration at various light intensity $\mathrm{I}=(9,18,30) \mathrm{mW} / \mathrm{cm}^{2}$, for curves $(1,2,3)$; also shown are the measured data of O'Brart et al [41], shown by blue bars.

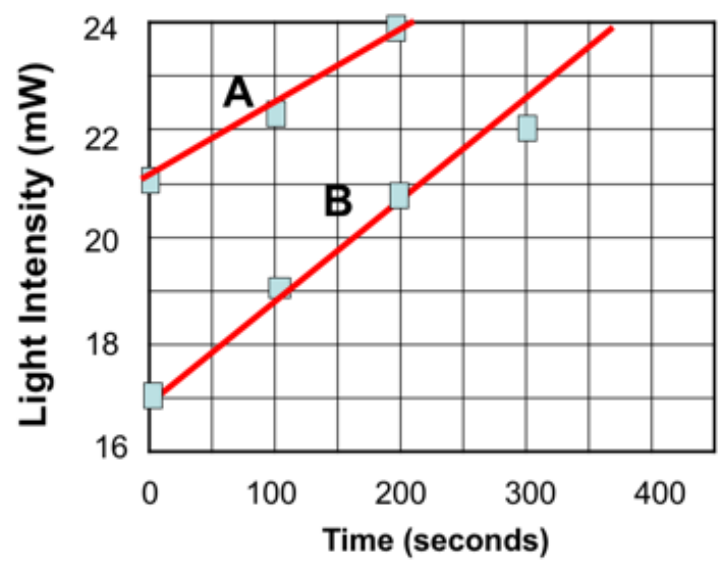

Fig. 7 Measurement of dynamic light intensity due to the RF (mixed with pure water) under UV irradiation at various RF concentrations of 0.0025 (curve A), and $0.005 \%$ (Curve B).

\subsection{Analysis of new protocol}


Fig. 8 Light intensity versus irradiation time $(\mathrm{t})$ based based on $\mathrm{BRL}$, or $\mathrm{I}_{0} \mathrm{t}=$ constant $=5.4$ $\mathrm{mJ} / \mathrm{cm}^{2}$, or $I_{0}=90 / t$, for $t$ in minutes and $I_{0}$ in $\mathrm{mJ} / \mathrm{cm}^{2}$ (shown in blue). The nonlinear law of Eq. (12.a) (shown in red dots) leads to $I_{0}=[45 / t]^{2}$, where we used a half-dose (or $2.7 \mathrm{~mJ} / \mathrm{cm}^{2}$ , based on the data of Hafez et al [42]), and corrected nonlinear law of $t=\mathrm{E}_{0} / \mathrm{l}_{0}^{0.5}$ versus the linear law of $B R L, t=E_{0} I_{0}$. We note that without the half-dose adjustment, the irradiation time $(t)$ in nonlinear law will be much longer than the BRL, and only slightly longer after the adjustment.

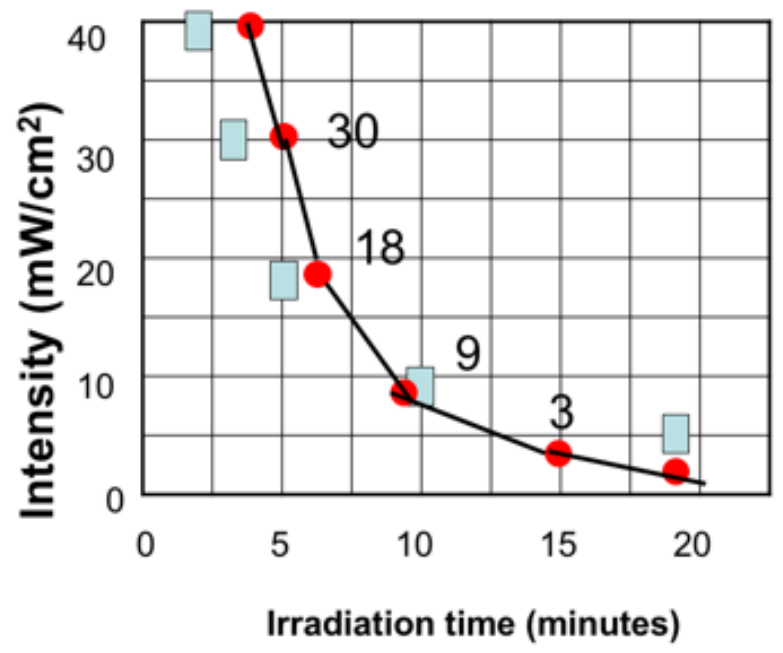

Fig. 8 Light intensity versus irradiation time (t) based on nonlinear law of Lin [ ], (shown in red dots), comparing to that based on BRL (shown in blue).

\subsection{Summary of up-dated CXL Features}

Factors influencing the CXL efficacy include: UV-A light intensity, dose, exposure time, mode of exposure (pulsed or $\mathrm{CW}$ ), riboflavin concentration, diffusion and drops pre-operation and interoperation administration, concentration of oxygen in the stromal tissue (pre-op and inter-op), and environmental conditions. The length of the riboflavin presoaking time and viscosity of the riboflavin film also affect the crosslink depth. From our analytic formulas, Eq. (4) to (11), and scaling laws, Eq. (110 to (13), the key features of type-I and type-II CXL are summarized and compared as follows:

(a) Type-I and type-II coexist in CXL, in the presence of oxygen. However, there is no type-II when oxygen is depleted or in a condition without oxygen.

(b) Type-I has two cases: case (i) with unimolecular termination, the radical $(R)$ and efficacy are a linear increasing function of blgC/G, but they are decreasing function of oxygen due to the $\mathrm{OIH}$ effect which reduces the radical $(R)$ and the efficacy, because $G$ is an increasing function of oxygen (or $X$ ), $G=G=k " X+K_{1}[A]$. In comparison, case (ii) for bimolecular termination, $\mathrm{R}$ is a nonlinear square-root function of $\left[\mathrm{K}_{3}(\mathrm{blgC})[\mathrm{A}]\right]^{0.5}$, but OlH plays no role.

(c) Type-II is oxygen mediated with efficacy proportional to the light dose and oxygen concentration, as shown by Eq. (7).

(d) The scaling laws of type-I efficacy given by, Eq. (12): $E_{0}\left[C_{0} / l_{0}\right]^{0.5}$ for transient state, and $\mathrm{Y}=\mathrm{m}^{\prime}\left[\mathrm{C}_{0} / \mathrm{l}_{0}\right]^{0.5}$ for steady state.

(e) The scaling laws of type-II efficacy given by, Eq. (13): $X^{\prime} t=C_{0} E_{0} X_{0}$ for transient state, and

(f) steady state $C E=1-\exp \left(-K X_{0}\right)$, which is almost independent to the light dose, or $R F$ concentration and it is solely depending on oxygen initial concentration $\left(X_{0}\right)$, given by a scaling law of $\mathrm{CE}=\mathrm{KX}$ (1- $\left.0.5 \mathrm{KX}_{0}+\mathrm{KX} / 6+\ldots\right)$.

(a) Oxygen is required for oxygen-mediated (OM) type-II but it is not required in in type-I. Therefore, type-II only plays a limited and transient state role for $t<t_{0}$, with $t_{0}$ being the 
depletion time of oxygen. In the transient stage (about 3 to 20 seconds), both type-I and type-Il coexist until the oxygen is depleted; then type-I dominates before the oxygen is resupplied or replenished.

(b) RF depletion in type-I is compensated by the RF regeneration term (RGE) which is a decreasing function of oxygen. For the case of perfect regeneration RF is a constant due to the catalytic cycle.

(c) Wernli et al [14] reported a sudden efficacy decrease at high light intensity (about 65 $\mathrm{mW} / \mathrm{cm}^{2}$ ), is also predicted by our formula, Eq. (8), and Fig 1, that the steady state efficacy is a decreasing function of light intensity, and a sudden drop is expected when the efficacy is below an efficacy threshold.

(d) The minimum corneal thickness $\left(Z^{*}\right)$ and the demarcation line depth (DLD), $Z^{\prime}$, are given by formula $Z^{*}=\left(1^{\prime} q^{\prime}\right) \ln \left(R^{\prime}\right)$, with $R^{\prime}=K E_{0} / E^{*}$; and $Z^{\prime}=\left(1 / q^{\prime}\right) \ln \left(R^{\prime}\right)$, with $R^{\prime}=K E_{0} / \ln E^{\prime}$, respectively, where both are increasing function of $\ln \left(E_{0}\right)$.

\section{CONCLUSION}

The controversial issues of $C X L$ are partially resolved via our analytic formulas, and compared with measurements. The roles of RF concentration and oxygen in the CXL efficacy could be analytically expressed. The scaling laws of type-I and type-II efficacy are different and given by analytic formulas. Our formulas also predict the maximum light intensity and the minimum corneal thickness, which are consistent with measurements.

\section{CONSENT}

It is not applicable.

\section{ETHICAL APPROVAL}

It is not applicable.

\section{COMPETING INTERESTS}

The author is the CEO of New Vision Inc. and has financial interest.

\section{ACKNOWLEDGEMENT}

The author likes to thank Kuo-Ti (Tony) Chen (at PhotoDynamic Corp, Taoyuan, Taiwan), and Jonathan Hu Yuan Kai (at Kaohsiung American School, Taiwan) for the assistance of computer drawings, and the measured data of Fig. 7, respectively.

\section{References}

1. Hafezi F and Randleman JB. editors. Corneal Collagen Cross-linking, second ed. Thorofare (NJ): SLACK; 2017.

2. Spoerl E, Huhle M, Seiler T. Induction of cross-links in corneal tissue. Exp Eye Res. 1998;66:97- 103.

3. Wollensak G, Spoerl E, Seiler T. Riboflavin/ultraviolet-a-induced collagen crosslinking for the treatment of keratoconus. Am J Ophthalmol. 2003;135:620-7.

4. Choi M, Kim J, Kim EK, Seo KY, Kim TI. Comparison of the conventional Dresden protocol and accelerated protocol with higher ultraviolet intensity in corneal collagen cross-linking for keratoconus. Cornea. 2017;36(5):523- 9.

5. Lang PZ, Hafezi NL, Khandelwal SS et al. Comparative Functional Outcomes After Corneal Crosslinking Using Standard, Accelerated, and Accelerated With Higher Total Fluence Protocols. Cornea 2019;38:433-441.

6. Bunsen RW, Roscoe HE. Photochemical researches-Part V. On the measurement of the chemical action of direct and diffuse sunlight. Proc R Soc Lond. 1862;12:306-12.

7. Lin JT. Analytic formulas on factors determining the safety and efficacy in UV-light sensitized corneal cross-linking. Invest Ophthalmol Vis Sci 2015; 56:5740-5741.

8. Lin JT. On the dynamic safety for cross linking in thin corneas (350-398 um) with extra protection under a contact lens. J Refract Sur 2015:31,7:495-496.

9. Lin JT, Cheng DC, Chang C, Yong Zhang. The new protocol and dynamic safety of UV-light activated corneal collagen cross-linking. Chinese J Optom Ophthalmol Vis Sci. 
2015;17:140-147

10. Lin JT. Combined analysis of safety and optimal efficacy in UV-light-activated corneal collagen crosslinking. Ophthalmology Research. 2016; 6(2):1-14.

11. Lin JT, Cheng DC. Modeling the efficacy profiles of UV-light activated corneal collagen crosslinking. PloS One. 2017;12:e0175002.

12. Lin JT. Efficacy and $Z^{*}$ formula for minimum corneal thickness in UV-light crosslinking. Cornea, 2017: 36:30-31.

13. Lin JT. Photochemical Kinetic modeling for oxygen-enhanced UV-light-activated corneal collagen crosslinking. Ophthalmology Research, 2017;7:1-8.

14. Lin JT. Efficacy S-formula and kinetics of oxygen-mediated (type-II) and non-oxygenmediated (type-I) corneal cross-linking. Ophthalmology Research. 2018; 8(1): 1-11.

15. Lin JT. A Critical Review on the Kinetics, Efficacy, Safety, Nonlinear Law and Optimal Protocols of Corneal Cross-linking. J Ophthalmology \& Visual Neuroscinece, 2018; 3:017.

16. Lin JT. A proposed concentration-controlled new protocol for optimal corneal crosslinking efficacy in the anterior stroma. Invest. Ophthalmol Vis Sci. 2018;59:431-432.

17. Lin JT. The role of stroma riboflavin concentration in the efficacy and depth of corneal crosslinking. Invest. Ophthalmol Vis Sci. 2018; 59:4449-4450.

18. Lin JT. Influencing factors relating the demarcation line depth and efficacy of corneal crosslinking. Invest Ophthalmol Vis Sci. 2018;59:5125-5126.

19. Lin JT, HW Liu, Chen KT, Cheng DC. Modeling the optimal conditions for improved efficacy and crosslink depth of photo-initiated polymerization. Polymers. 2019, 11, 217; doi:10.3390/polym11020217.

20. Lin JT. Modeling a new strategy and influencing factors for improved efficacy of accelerated corneal crosslinking. J Cataract Refract Surg, 2019, 45, 527-529.

21. Lin JT, Chen KT, Cheng DC, Liu HW. Modeling the efficacy of radical-mediated photopolymerization: the role of oxygen inhibition, viscosity and induction time. Front. Chem. 2019, 7:760. doi: 10.3389/fchem.2019.00760.

22. Lin JT. Up-dated the Critical Issues of Corneal Cross-linking (type-I and II): safety dose for ultra-thin cornea, demarcation line depth and the role of oxygen. Ophthalmology Res. 2021;4(1);1-7.

23. Sheng SF, Lin JT. Critical analysis of corneal cross-linking(Part-I): formulas for efficacy, safety dose, minimum thickness, demarcation line depth, maximum light intensity, and the role of oxygen. Ophthalmology Research, An International Journal, 2021.14:29-41; DOI: 10.9734/OR/2021/v14i430200.

24. Kamaev P, Friedman MD, Sherr E, Muller D. Cornea photochemical kinetics of corneal cross-linking with riboflavin. Vis. Sci. 2012;53:2360-2367.

25. Schumacher S, Mrochen M, Wernli J, Bueeler M, Seiler T. Optimization model for UVriboflavin corneal cross-linking. Invest Opthamol Vis Sci. 2012; 53:762-769.

26. Semchishen A, Mrochen A, Semchishen V. Model for optimization of the UV-A/Riboflavin strengthening (cross-linking) of the cornea: percolation threshold. Photochemistry and photobiology, 2015; 91:1403-1411.

27. Kling S, Hafezi F. An algorithm to predict the biomechanical stiffening effect in corneal cross-linking. J Refract Surg 2017; 32:128-136. doi:10.3928/1081597X-20161206-01.

28. Wollensak G, Spoerl E, Wilsch M, Seiler T. Endothelial cell damage after riboflavinultraviolet-A treatment in the rabbit. J Cataract Refract Surg 2003;29:1786-90.

29. Mooren P, Gobin L, Bostan N et al. Evaluation of UVA cytotoxicity for human endothelium in an ex vivo corneal cross-linking experimental setting. J Refract Surg. 2016;32:4-46.

30. Wernli J, Schumacher S, Spoerl E, Mrochen M. The efficacy of corneal cross-linking shows a sudden decrease with very high intensity UV light and short treatment time. Invest Ophthalmol Vis Sci. 2013;54:1176-80.

31. Mazzotta C, Sgheri A, Bagaglia SA, et al. Customized corneal crosslinking for treatment of progressive keratoconus: Clinical and OCT outcomes using a transepithelial approach with supplemental oxygen. J Surg. $2020 \quad$ Dec;46(12):1582-1587. doi: 10.1097/j.jcrs.0000000000000347.

32. Sachdev GS, Ramamurthy S, Dandapani R. Photorefractive intrastromal corneal crosslinking for treatment of low myopia: clinical outcomes using the transepithelial 
approach with supplemental oxygen. J Cataract Refract Surg 2020;46:428-433

33. Kling S, Richoz O, Hammer A, et al. Increased Biomechanical Efficacy of Corneal Crosslinking in Thin Corneas Due to Higher Oxygen Availability. J Refract Surg 2015;31:840-6.

34. Torres-Netto EA, Kling $\mathrm{S}$, Hafezi $\mathrm{N}$ et al. Oxygen Diffusion May Limit the Biomechanical Effectiveness of lontophoresis-Assisted Transepithelial Corneal Cross-linking. J Refract Surg 2018;34:768-774.

35. Mazzotta C, Traversi C, Caragiuli S, Rechichi M. Pulsed vs. continuous light accelerated corneal collagen crosslinking:In vivo qualitative investigation by confocal microscopy and corneal OCT. Eye (Lond) 2014;28:1179-83.

36. Mazzotta C, Bagaglia SA, Vinciguerra R, Ferrise M, Vinciguerra P. Enhanced-fluence pulsed-light iontophoresis corneal cross-linking: 1-year morphological and clinical results. J Refract Surg 2018;34:438-444

37. Mazzotta C, Bagaglia SA, Sgheri A et al.. lontophoresis corneal cross-linking with enhanced fluence and pulsed UV-A light: 3-year clinical results. J Refract Surg 2020;36:286-292.

38. Kanellopoulos AJ, Dupps WJ, Seven I, Asimellis G. Toric Topographically Customized Transepithelial, Pulsed, Very High-Fluence, Higher Energy and Higher Riboflavin Concentration Collagen Cross-Linking in Keratoconus. Case Report Ophthalmol 2014;5:172-180.

39. Seiler TG, Batista A, Frueh BE, Koenig K. Riboflavin Concentrations at the Endothelium During Corneal Cross-Linking in Humans. Invest Ophthalmol Vis Sci 2019;60:2140-2145.

40. Seiler T, Batista, Frueh BE, Koenig K. Riboflavin Concentrations at the Endothelium During Corneal Cross- Linking in Humans. Invest. Ophthalmol Vis Sci. 2019;60:2140-2145.

41. O'Brart NAL, O'Brart DPS, Aldahlawi et al, An Investigation of the effects of riboflavin concentration on the efficacy of corneal cross-Linking using an enzymatic resistance model in porcine corneas. Invest. Ophthalmol Vis Sci. 2018; 59: 1058-1065. doi:10.1167/iovs.1722994.

42. Kling S, Hafezi F. Biomechanical stiffening: slow low-irradiance corneal crosslinking versus the standard Dresden protocol. J Cataract Refract Surg 2017; 43:975-979 Mazzotta C, Traversi C, Caragiuli S, Rechichi M. Pulsed vs. continuous light accelerated corneal collagen crosslinking:In vivo qualitative investigation by confocal microscopy and corneal OCT. Eye (Lond) 2014;28:1179-83.

43. Hafezi F, Kling S, Gilardoni F, et al. Individualized corneal cross-linking with riboflavin and UV-A in ultra-thin corneas: the sub400 protocol. Am J Ophthalmol. 2021;224:133-142. doi:10.1016/j.ajo.2020.12.011.

44. Mazzotta C, Riomani A, Burroni A. Pachymetry-based Accelerated Cross-linking: The "M Nomogram" for Standardized Treatment of All-thickness Progressive Ectatic Corneas. Int K Kerat Ect Corn Dis 2019;7(2):137-144. 